\title{
Les primes d'objectifs vecteurs de signaux en fonction de leur montant
}

\section{Maryline Bourdil}

\section{(2) OpenEdition}

12 Journals

Édition électronique

URL : http://journals.openedition.org/communicationorganisation/3762

DOI : 10.4000/communicationorganisation.3762

ISSN : $1775-3546$

Éditeur

Presses universitaires de Bordeaux

\section{Édition imprimée}

Date de publication : 1 juin 2012

Pagination : 115-129

ISBN : 978-2-86781-821-9

ISSN : $1168-5549$

\section{Référence électronique}

Maryline Bourdil, «Les primes d'objectifs vecteurs de signaux en fonction de leur montant ». Communication et organisation [En ligne], 41 | 2012, mis en ligne le 01 juin 2015, consulté le 19 avril 2019. URL : http://journals.openedition.org/communicationorganisation/3762 ; DOI : 10.4000/ communicationorganisation.3762 


\title{
Les primes d'objectifs vecteurs de signaux en fonction de leur montant
}

\author{
Morụline Bourdil
}

La plupart des téléconseillers ont dans la réalisation de leur métier deux principaux rôles : vendre des produits, mais également satisfaire leurs clients (Buscatto, 2002 ; Clergeau, Marciniak et Rowe, 2002 ; Perrier, 2002 ; Pichault et Zune, 2000). Ces deux rôles, que sont la vente, et la satisfaction client semblent difficilement compatibles (Honneycut et al, 2001 ; Kalra et al, 2003 ; Lavorata, 2005 ; Menguc et Barker, 2003 ; Sharma et Sarel, 1995 ; Widmier, 2002). Quantité et qualité seraient plus exclusives que conciliables.

Il s'agit dans ce travail de recherche d'étudier l'impact de la part de la prime d'objectifs dans le salaire de base sur l'ambiguïté de rôle. Il semble que non seulement les personnes mais aussi les outils tels qu'une prime d'objectifs peuvent envoyer des signaux. Quel signal la prime d'objectifs fait-elle passer aux téléconseillers concernant leur rôle?

Il est apparu essentiel de rapporter le montant de la prime d'objectifs au salaire de base. Si le salaire de base n'est pas le même, une prime d'un montant similaire, n'aura pas le même impact. D'ailleurs la prise en compte de la variable " part de la prime d'objectifs dans le salaire de base » au lieu des variables « présence ou absence de la prime d'objectifs » ou « montant de la prime d'objectifs » constitue un des intérêts de cette étude.

Dans un premier temps, le cadre théorique qui est celui de la théorie des rôles (Kahn et al, 1964) et de la théorie du signal sera présenté. Par la suite l'étude empirique, sa méthodologie, les résultats obtenus seront exposés. Pour finir les implications managériales seront présentées.

\section{Revue de littérature}

Le cadre théorique retenu est celui de la théorie des rôles (Kahn et al, 1964) et de la théorie du signal. Les études empiriques utiles à la formulation de l'hypothèse que nous allons tester seront passées en revue.

1 Maryline Bourdil est professeur assistant en gestion des resources humaines au GSCM-Montpellier Business School; m.bourdil@supco-montpellier.fr 


\section{I.1 L'ambiguïté de rôle (Khan et al, 1964)}

Préalablement à la définition du concept d'ambiguïté de rôle, il convient de mentionner quelques éléments. La personne focale est celle dont le rôle fait l'objet de l'attention du chercheur. Elle est aussi appelée personne cible. Les émetteurs sont les personnes qui entourent la personne cible et qui formulent, de façon plus ou moins explicite des attentes à l'égard de la personne focale. Il peut s'agir des pairs comme de supérieurs hiérarchiques, voire de clients. Dans cette définition originelle, il est possible de voir que l'émetteur est une personne.

Or, ne peut-on pas considérer qu'un outil, en l'occurrence ici, une prime d'objectifs soit émetteur d'attentes?

L'ambiguité de rôle est un déficit d'information quant à l'existence ou la clarté des attentes concernant son rôle (Rizzo et al, 1970). L'ambiguïté de rôle est par conséquent la situation dans laquelle l'individu ne possède pas les informations nécessaires concernant l'exécution de sa tâche ou lorsque les informations ne sont pas claires, voire parfois se contredisent (Rizzo et al, 1970).

Kahn et al (1964) ont distingué : (1) l'ambiguïté de rôle objective, résultant du fonctionnement de l'organisation et des conditions réelles de l'environnement, (2) l'ambiguité de rôle subjective, liée à l'état psychologique de la personne.

L'ambiguïté de rôle objective liée à la tâche résulte d'un manque d'information concernant la définition du poste, ses objectifs et les moyens prévus pour l'améliorer. Trois formes spécifiques correspondant à ce type d'ambiguiité de rôle ont été définies et relèvent de trois problèmes centraux. (1) L'ambiguité de rôle quant au contenu du poste en fonction de ce qui est demandé: incertitude concernant les missions et l'étendue des responsabilités. Quelles sont les missions et les responsabilités de l'individu en place dans le poste ? (2) L'ambigü̈té liée à la façon de mener à bien sa tâche et ses responsabilités: incertitude concernant les comportements nécessaires pour remplir ses tâches et ses responsabilités. Comment l'individu doit-il procéder pour accomplir efficacement ses tâches et répondre adéquatement à ses responsabilités ? (3) L'ambiguïté de rôle quant aux attentes du (ou des) sous-système(s): incertitude concernant les attentes des émetteurs. A noter que si les primes d'objectifs sont sources d'ambiguïté de rôle nous nous situons ici.

Quant à l'ambiguité de rôle subjective il s'agit de l'ambiguité provenant des conséquences des comportements et des incertitudes concernant les effets des actions de la personne focale sur son bien-être, sur le système de rôle et sur l'organisation dans son ensemble.

\section{I.2 Le métier de téléconseiller : des rôles parfois difficilement conciliables}

Selon Buscatto (2002), Clergeau, Marciniak et Rowe (2002), Perrier (2002), Pichault et Zune (2000), la plupart des téléconseillers auraient deux 
principaux rôles que sont la vente, et le service client à travers l'assurance d'une certaine qualité.

Les centres d'appels poursuivent un double objectif : la productivité et la satisfaction client (Clergeau, Marciniak et Rowe, 2002). Plus précisément, il s'agit de (1) répondre ou émettre un maximum d'appels par unité de temps, (2) développer le chiffre d'affaires en fidélisant la clientèle par l'offre d'un service de qualité, et en accédant rapidement à une clientèle potentielle ciblée, (Buscatto, 2002 ; Perrier, 2002 ; Pichault et Zune, 2000).

Les deux logiques sont-elles compatibles ? La productivité ne l'emporterait-elle généralement pas?

Au vu des travaux d'auteurs tels que Brown et Maxwell (2002), Di Ruzza et Franciosi, (2002), on pourrait effectivement penser que la productivité serait le maître mot. Les centres d'appels sont en effet présentés comme " aliénants ". Les cadences et rendement sont fixés par des normes strictes (Di Ruzza et Franciosi, 2002). Selon Brown et Maxwell (2002), cette image productiviste peut être attribuée en partie au générateur automatique d'appels, système qui compose automatiquement, par anticipation, les numéros de téléphone listés dans les fichiers du centre d'appels.

\section{I.3 Résultats empiriques}

Il ressort des études quantitatives d'Agarwal (1996) et de Jaworski et Mac Innis (1989), que le contrôle des résultats engendre des problèmes d'ambiguïté de rôle. Or, toute prime d'objectifs est liée à un système d'évaluation, de contrôle des résultats.

Rouzies et Besson (1998) concluent, suite à leur étude exploratoire, que les systèmes hybrides qui consistent à attribuer aux employés un salaire fixe plus des commissions ou des primes d'objectifs sont sources d'ambiguïté de rôle.

Au vu des résultats des études empiriques (Agarwal, 1996 ; Jaworski et Mac Innis, 1989 ; Rouzies et Besson, 1998), il semblerait que les primes d'objectifs soient susceptibles de générer de l'ambiguité de rôle.

Maintenant, si est considéré le montant de la prime d'objectifs, et plus précisément, la part de la prime d'objectifs dans le salaire de base, quelle est l'importance de l'impact sur l'ambiguïté de rôle?

Autrement dit l'ambiguïté de rôle varie-t'elle avec la part de la prime d'objectifs dans le salaire de base ?

\section{I.4 La théorie du signal}

Un signal est une information donnée par un émetteur. Ce signal aide le récepteur à prendre une décision.

Spence a écrit un article reconnu dans lequel est mobilisée la théorie du signal (1973). Cet auteur a appliqué la théorie du signal à l'éducation supérieure et trouvé que le niveau de diplôme envoie un signal au recruteur 
durant le processus de recrutement. Le diplôme prouve la compétence du candidat au recruteur.

Karasek and Bryant (2011) soulignent que "des signaux sont partout autour de nous, ce, tous les jours".

Quel signal la prime d'objectifs envoie-t-elle en fonction de la part qu'elle représente dans le salaire de base?

Partant du principe que les primes d'objectifs viennent récompenser l'atteinte d'objectifs qui sont principalement quantitatifs (exemple: nombre de ventes réalisées), trois cas de figure peuvent être distingués et par conséquent trois propositions peuvent être formulées (cf tableau 1 ) :

1. Une absence de prime d'objectifs signifierait clairement que «la vente n'est pas primordiale».

2. Une prime moyenne voudrait dire «la vente n'est pas primordiale, mais il faut vendre ». Ici un commentaire s'impose. Nous voyons bien que le signal envoyé par la prime d'objectifs n'est pas clair, ambigu. Le risque d'ambiguïté de rôle chez les téléconseillers est alors présent.

3. Lorsque la prime est forte, le signal émis est alors clair : « il faut vendre».

\begin{tabular}{|l|l|}
\hline \multicolumn{1}{|c|}{ Montant de la prime d'objectifs } & \multicolumn{1}{c|}{$\begin{array}{c}\text { Signal envoyé par la prime d'objectifs } \\
\text { concernant le rôle de vente }\end{array}$} \\
\hline Pas de prime & "La vente n'est pas primordiale » \\
\hline Prime moyenne & $\begin{array}{l}\text { "La vente n'est pas primordiale mais il faut } \\
\text { vendre» }\end{array}$ \\
\hline Prime forte & "Il faut vendre» \\
\hline
\end{tabular}

Tableau 1 : Signal envoyé par la prime d'objectifs concernant le rôle de vente du téléconseiller

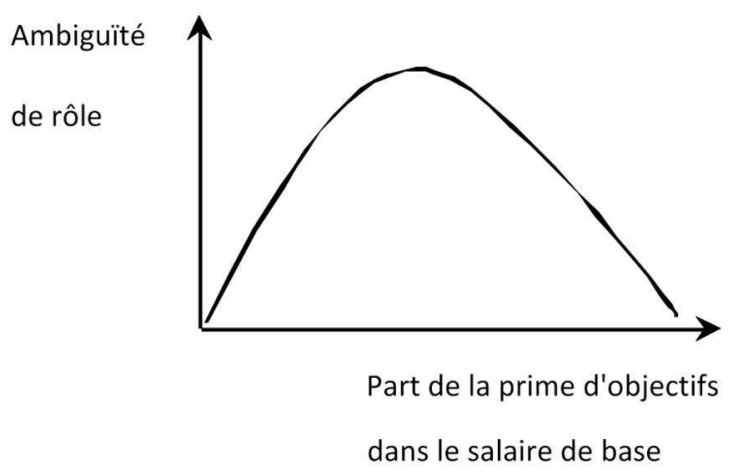

Figure 1 : Lien entre la part de la prime d'objectifs dans le salaire de base et l'ambiguîté de rôle 
Ainsi il est légitime de penser qu'il devrait y avoir une relation en U inversé entre la part de la prime d'objectifs dans le salaire de base et l'ambiguïté de rôle (cf. figure 1).

L'ambiguïté de rôle sera moins élevée lorsqu'il n'y a pas de prime d'objectifs ou lorsque celle-ci est forte. L'ambiguïté de rôle sera plus élevée quand la prime est modérée.

L'hypothèse ci-dessous est ainsi formulée :

$\mathrm{H}:$ La part de la prime d'objectifs dans le salaire de base a un impact de type non linéaire sur l'ambiguité de rôle.

La question est maintenant également de savoir à partir de quels seuils les effets de la prime d'objectifs sont observés?

\section{Etude empirique}

\section{II.1 Méthodologie}

Une étude exploratoire a été menée auprès de quinze téléconseillers et de cinq responsables d'équipe de téléconseillers dans un même centre d'appels (cf annexe 1). Les résultats des entretiens semi-directifs menés auprès de ces vingt individus seront utilisés lors de la phase des implications managériales.

L'échantillon de l'étude quantitative est composé de cent dix-huit téléconseillers issus de sept centres d'appels (produits surgelés, compléments alimentaires, banque, télécommunications, accessoires et véhicules de loisirs) dont celui où a eu lieu l'étude exploratoire. 74,1\% sont des femmes, $56 \%$ ont moins de trente ans et $12,9 \%$ ont plus de quarante-six ans. $67,7 \%$ des téléconseillers ont moins de trois ans d'ancienneté.

Concernant la mesure des variables elle s'est faite de la manière suivante. (1) Concernant la variable " part de la prime d'objectifs dans le salaire de base ", deux questions, l'une relative au montant de la prime moyenne d'objectifs et l'autre au montant du salaire fixe net ont été posées (cf annexe 2). A partir de ces données, la part de la prime d'objectifs dans le salaire de base a été calculée. Les tranches de la part de la prime d'objectifs dans le salaire de base $(0-10 \%, 11-20 \%, 21-30 \%, 31-70 \%, 71-100 \%)$ ont été retenues afin que la répartition dans chacune soit au minimum de 11 individus. (2) Pour mesurer l'ambiguïté de rôle l'échelle de mesure retenue est celle de Rizzo et al (1970) (cf annexe 2). Le coefficient alpha de l'échelle de l'ambiguïté de rôle est 0,8255 après épuration et le pourcentage de variance expliquée de 74,653.

L'hypothèse a été testée via une analyse de variance (ANOVA); test ici le plus approprié puisque nous cherchons une relation non linéaire (Evrard et al., 2003). Ainsi pour tester la possibilité d'une relation non linéaire entre la part de la prime d'objectifs dans le salaire de base et l'ambiguïté de rôle le logiciel SPSS a été utilisé. 


\section{II.2 Résultats}

Avant de réaliser l'analyse de variance (ANOVA) trois valeurs éloignées et deux valeurs extrêmes ont été supprimées (cf graphique 1). Ces valeurs correspondent aux réponses des téléconseillers dont l'ambiguïté de rôle diffère sensiblement de l'ambiguïté de rôle moyenne des téléconseillers.

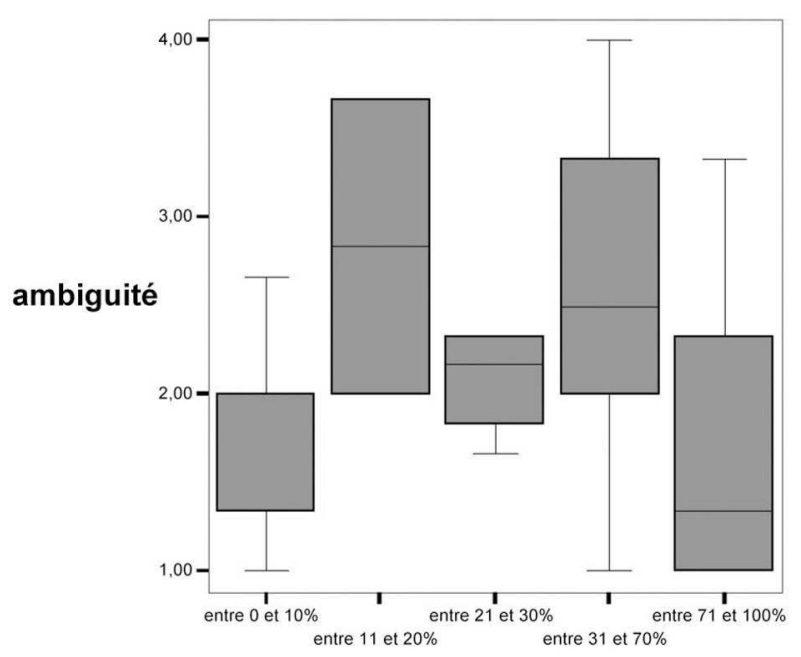

POURCENT

Graphique 1 : Boîte à moustaches de l'ambiguité de rôle en fonction de la part de la prime d'objectifs dans le salaire de base après suppression des valeurs éloignées et extrêmes

L'analyse de la variance (ANOVA) dévoile une relation significative entre l'ambiguité de rôle et la prime d'objectifs dans le salaire de base $(\rho<0.05$; cf. tableau 2).

\begin{tabular}{|l|c|c|c|c|c|}
\hline & $\begin{array}{c}\text { Somme des } \\
\text { carrés }\end{array}$ & ddl & $\begin{array}{c}\text { Moyenne } \\
\text { des carrés }\end{array}$ & F & Signification \\
\hline Inter-groupes & 6,177 & 4 & 1,544 & 3,170 & $0,021^{* *}$ \\
Intra-groupes & 24,360 & 50 & 0,487 & & \\
Total & 30,537 & 54 & & & \\
\hline
\end{tabular}

**: la différence de moyenne est significative au niveau 0,05 (test de Fisher unilatéral)

Tableau 2 : ANOVA, part de la prime d'objectifs dans le salaire de base et ambiguiité de rôle

Si l'on regarde les moyennes de l'ambiguïté de rôle, il semble que l'ambiguïté de rôle soit plus forte lorsque la part de la prime d'objectifs dans le salaire est comprise entre 10 et $70 \%$ (cf tableau 3). 


\begin{tabular}{|c|c|c|c|}
\hline Pourcentage & Moyenne & n & Ecart-type \\
\hline Entre 0 et $10 \%$ & 2,0303 & 33 & 0,85539 \\
\hline Entre 11 et $20 \%$ & 2,8333 & 2 & 1,17851 \\
\hline Entre 21 et $30 \%$ & 2,5333 & 5 & 1,04350 \\
\hline Entre 31 et $70 \%$ & 2,5833 & 8 & 0,97183 \\
\hline Entre 71 et $100 \%$ & 1,7222 & 12 & 0,87425 \\
\hline
\end{tabular}

Tableau 3 : Moyennes de l'ambiguïté de rôle en fonction de la part de la prime d'objectifs dans le salaire de base

Il y aurait par conséquent une relation en $U$ inversé entre la part de la prime d'objectifs dans le salaire de base et l'ambiguïté de rôle. Cette relation est d'ailleurs visible sur le graphique retraçant le lien entre la part de la prime d'objectifs dans le salaire de base et l'ambiguïté de rôle (cf graphique 2).

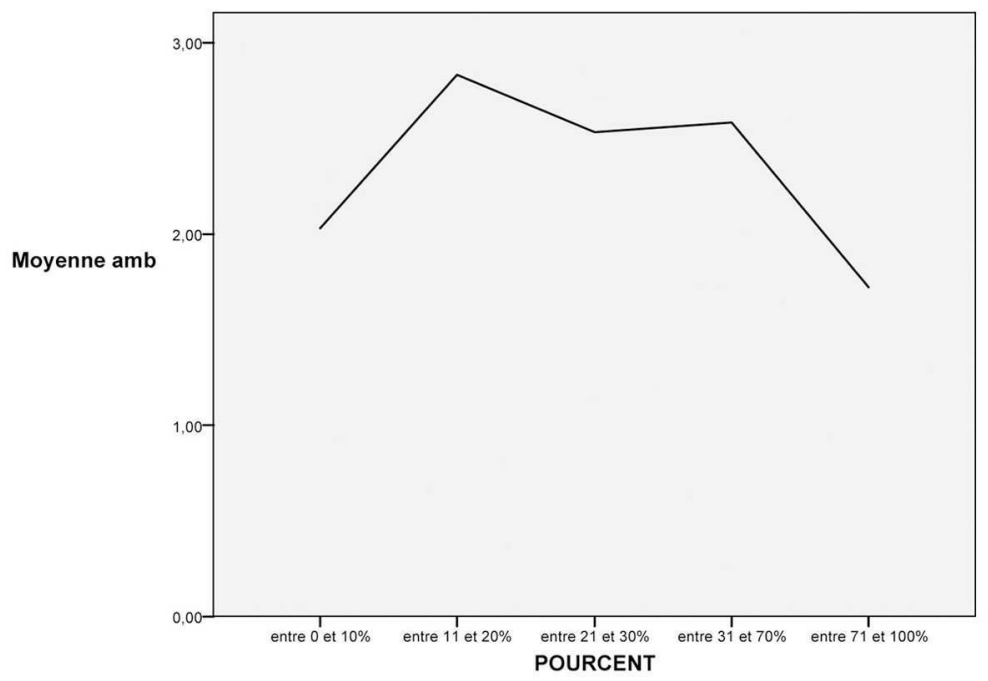

Graphique 2 : Lien entre la part de la prime d'objectifs dans le salaire de base et l'ambiguiité de rôle

Il ressort du test de Tukey que lorsque la part de la prime d'objectifs est comprise entre 0-10\% et entre 31-70\%, la moyenne de l'ambiguité de rôle est significativement différente (cf tableau 4). 


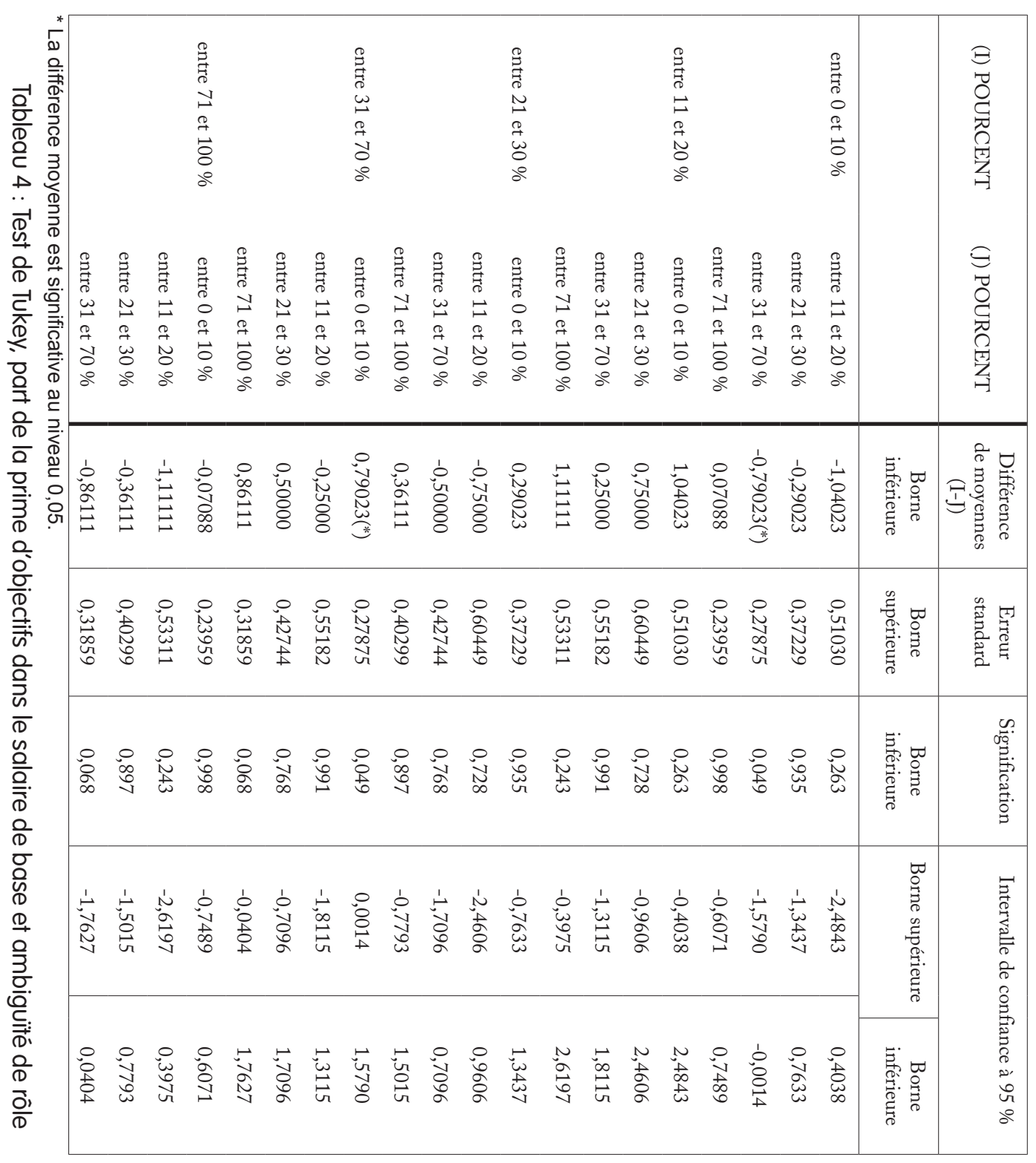

L'hypothèse $\mathrm{H}$ selon laquelle la part de la prime d'objectifs dans le salaire de base a un impact de type non linéaire sur l'ambiguité de rôle est acceptée.

Il y a une relation en U inversé entre la part de la prime d'objectifs dans le salaire de base et l'ambiguïté de rôle. 


\section{II.3 Implications managériales}

Lorsque la part de la prime d'objectifs dans le salaire de base est comprise entre 11 et $70 \%$, mais surtout entre 11 et $20 \%$, le signal envoyé par la prime d'objectifs n'est pas clair. Les téléconseillers subissent la plus forte ambiguité de rôle.

Par contre en dessous de $10 \%$, et au dessus de $70 \%$, le signal est clair. L'ambiguïté de rôle est ainsi plus faible, surtout lorsque la part de la prime d'objectifs dans le salaire de base est supérieure à $70 \%$.

Ainsi les implications managériales peuvent être les suivantes. Pour réduire l'ambiguïté de rôle, qui est une source de stress : (a) il est préférable de ne pas mettre en place de prime d'objectifs ou une prime faible (0-10\%), ou à l'inverse, (b) une prime d'objectifs élevée (plus de $70 \%$ ). Toutefois il semble important d'attirer l'attention sur les dangers d'une prime d'objectifs élevée (Honneycut et al., 2001; Kalra et al., 2003; Lavorata, 2005; Sharma and Sarel, 1995; Widmier, 2002). Le signal est certes clair : vendre est plus important que la satisfaction clients. Mais, qu'en est-il de l'effet pervers d'une telle prime sur les comportements vis-à-vis de la clientèle ?

Un responsable d'équipe interviewé souligne le danger du déplafonnement de la prime d'objectifs sur les comportements vis-à-vis des clients. Il parle de «ventes abusives ». Plus les téléconseillers vendent, plus ils sont rémunérés, ceci sans aucune limite. Tous les moyens sont alors bons dans un seul but qui est celui de vendre.

"[...] on peut très bien s'imaginer la dérive, c'est-à-dire le téléconseiller qui vend abusivement, mais ça c'est à nous de vérifier que ce n'est pas le cas, et qui finisse par vendre mal finalement pour vendre plus mais pas forcément mieux, dans le sens de bien adapté. Voilà donc pour moi le plafond est quand même nécessaire. Tel qu'il était défini en 2003, c'était un plafond à 540 euros, c'était peut être pas suffisant mais de là à déplafonner, c'est peut être, trop risqué. Il faut qu'il soit fonction du salaire fixe en fait ». (Interviewé 18)

Ce même responsable d'équipe préconise un plafond de l'ordre de 30 $40 \%$. Selon lui, afin d'éviter les effets pervers de la prime d'objectifs sur les comportements vis-à-vis de la clientèle, le montant maximal des primes d'objectifs ne devrait pas dépasser 30 à $40 \%$ du salaire de base. Or, à l'heure actuelle, certains téléconseillers doublent parfois leur salaire de base.

"Il y a peut être eu des dangers liés au fait qu'il y ait eu ce déplafonnement de PVV parce que peut - être qu'on arrive aujourd'hui à des extrêmes sur des performances. Peut - être faudrait-il imaginer un plafond, je ne sais pas. Il ne faudrait pas que cette PVV dépasse un certain montant de la rémunération fixe, c'est mon point de vue. Maintenant, c'est bien qu'elle soit quand même conséquente. Donc qu'elle soit égale à $30 \%, 40 \%$ du salaire fixe ça me paraît raisonnable, qu'elle soit supérieure à ce montant là, ça me paraît déraisonnable ». (Interviewé 18) 
Un téléconseiller souligne également le fait que parfois certains téléconseillers vont placer pour placer, ceci pour réaliser à tout prix des ventes.

Ce même téléconseiller souligne les problèmes que cela pose en terme de satisfaction client et d'image de l'entreprise.

"[...] il y a quand même une image de marque dans notre entreprise, c'est comme ça. Bon il y en a qui au niveau de la concurrence ont été condamnés, ils font des choses qui ne sont pas normales, mais notre entreprise, c'est une maison où on ne fait pas n'importe quoi, on a une image de marque quand même et voilà, on en vient aussi à faire n'importe quoi, donc ça craint. [...] Si quelqu'un n'atteint jamais ses chiffres, au bout d'un moment, il va peutêtre être convoqué, donc c'est pas bon, il faut atteindre le minimum quand même, donc c'est vrai qu'on est pris entre les deux ». (Interviewé 7)

"Soit on fait ce que l'entreprise dit, soit on fait ce que le client demande. Il ne faut pas stresser les gens pour qu'ils fassent plus et moins bien ». (Interviewé 7)

Ces verbatims corroborent les résultats d'études précédentes dont les auteurs concluent que les rémunérations liées au nombre de ventes engendrent des comportements néfastes vis-à-vis de la clientèle (Honneycut et al, 2001 ; Kalra et al, 2003 ; Lavorata, 2005 ; Sharma et Sarel, 1995 ; Widmier, 2002). En outre, Menguc et Barker (2003) ont montré que la rémunération totale des vendeurs est négativement et significativement liée à la satisfaction des clients.

Toujours concernant les comportements vis-à-vis de la clientèle, il est ici intéressant de souligner que d'après les résultats de l'étude empirique de Guery et al (2003) réalisée dans la même entreprise que celle ayant fait l'objet de notre étude exploratoire, ce problème de ventes parfois inadaptées existait auparavant, c'est-à-dire avant la mise en place d'une prime d'objectifs. A noter aussi que même s'il n'y avait à ce moment là pas de primes d'objectifs, il y avait tout de même en place un système de management par objectifs (objectifs à atteindre et contrôle des résultats).

Une question essentielle maintenant se pose. Le problème de ventes inadaptées a-t-il été accentué dans cette entreprise par l'introduction d'une prime d'objectifs ? Il se pourrait en effet que l'adjonction d'une récompense monétaire renforce l'impact du management par objectifs.

\section{Conclusion}

Si référence est faite uniquement à la théorie des rôles, l'émetteur est une personne. Pour cette raison, il est intéressant de mobiliser également la théorie du signal. Un outil tel qu'une prime d'objectifs, et non seulement une personne, peut envoyer un signal.

De plus, nous nous sommes interrogés sur la nature des signaux envoyés par les primes d'objectifs et leur compatibilité. Trois propositions ont 
été émises dans ce sens ${ }^{2}$ (pas de prime $\rightarrow$ "La vente n'est pas primordiale ", prime moyenne $\rightarrow$ "La vente n'est pas primordiale mais il faut vendre", prime forte $\rightarrow$ "Il faut vendre»).

A partir de ces propositions, l'hypothèse d'une relation non linéaire entre le montant de la prime d'objectifs dans le salaire de base et l'ambiguïté de rôle a été formulée. Nous avons testé l'hypothèse sur un échantillon de cent dix huit téléconseillers de sept centres d'appels. Il convient de reconnaître que la taille de l'échantillon constitue une limite de notre étude. A noter toutefois, les difficultés d'accès au terrain des centres d'appels étant donné leur mauvaise image.

Le principal résultat est que la relation entre la part de la prime d'objectifs dans le salaire de base et l'ambiguïté de rôle est en U inversé. L'hypothèse est ainsi validée. L'autre résultat important de cette étude est l'identification des seuils à partir desquels l'impact de la part de la prime d'objectifs dans le salaire de base sur l'ambiguïté de rôle varie. L'ambiguïté de rôle est forte lorsque la part de la prime d'objectifs dans le salaire de base est comprise entre 11 et $70 \%$. Par contre, au dessous de $10 \%$ et en dessus de $70 \%$, le signal est clair. L'ambiguité de rôle est ainsi plus faible, surtout lorsque la part de la prime d'objectifs dans le salaire de base est supérieure à $70 \%$. Dans ce cas le rôle le plus important est la vente.

Les managers, les entreprises qui utilisent des primes d'objectifs devraient retenir ceci : pour réduire l'ambiguïté de rôle qui est une source de stress, il vaut mieux ne pas avoir du tout de prime d'objectifs ou une prime d'objectifs faible (0-10\%), ou au contraire, une prime d'objectifs élevée (supérieure à $70 \%)$. Mais il semble important d'attirer l'attention sur les dangers d'une prime d'objectifs élevée notamment en terme de comportements vis-à-vis de la clientèle.

\section{BIBLIOGRAPHIE}

AGARWAL S., "Consequences of marketing controls among sales and non sales marketing personnel », Industrial Marketing Management, 25, 1996, p. 411-420.

BROWN G., MAXWELL G., "Customer Service in UK call centres organisational perspectives and employee perceptions ", Journal of Retailing and Consumer Services, 9(6), 2002, p. 309-316.

BUSCATTO M., "Les centres d'appels, usines modernes ? Les rationalisations paradoxales de la relation téléphonique », Sociologie du Travail, 44, 2002, p. 99-117.

CLERGEAU C., MARCINIAK R., ROWE F., « La performance et l'efficacité des centres d'appels : le cas des centres de réception d'appels ", $1^{\circ}$ journée nantaise de recherche sur le e-marketing, 2002, p. 31-55.

2 Cf. Tableau 1 


\section{$C \& O n^{\circ} 41$}

DI RUZZA R., FRANCIOSI C., Situations de travail dans les centres d'appels téléphoniques, Convention PESETT-CAT, Programme ACI Travail, ministère de la Recherche, 2 vol, avril, 2002.

GUERY L., MERCIER E., MOTTAY D., SCHMIDT G. (dir), TIC, flexibilité, transformations du travail et performances: places et rôles de la fonction $\mathrm{RH}$; le cas de France Télécom, DARES, Ministères des affaires sociales, du travail et de la solidarité, 2003.

HONEYCUTT E. D., GLASSMAN M., ZUGELDER M. T., KARANDE K., "Determinants of ethical behavior: a study of autosalespeople ", Journal of Business Ethics, 32, 2001, p. 69-79.

JAWORSKI B. J. et MC INNIS, « Marketing Jobs and Management Controls : Toward a Framework ", Journal of Marketing Research, 26 (4), 1989, p. 406-419.

KAHN R. L., WOLFE D. M., QUINN R. P., SNOECK J. D., ROSENTHAL R. A., Organizational stress: studies in role conflict and ambiguity, New York, John Wiley, 1964.

KARASEK R., BRYANT P., « Signaling theory: past, present, and future », Proceedings of the Academy of Strategic Management, 10(1), 2011, p. 27-32.

LAVORATA L., "Lien entre climat éthique et comportements éthique du vendeur : rôle déterminant du management commercial : une application en B to B ", Cahier de Recherche Université Paris XII, Institut de recherche en Gestion, 2005.

MENGUC B., BAKER T., "The performance effects of outcome-based incentive pay plans on sales organizations: a contextual analysis ", Journal of Personal Selling Management, 23 (4), 2003, p. 341-358.

PERRIER P., « Centres d'appels : la GRH confrontée à l'industrialisation des services, Etudes », Entreprise et Personnel, n’218, 2002.

PICHAULT F., ZUNE F., «Une figure de la déréglementation du marché du travail : le cas des centres d'appels ", Revue Management et Conjoncture Sociale, n580, 2000.

RIZZO J., HOUSE R. et LIRTZMAN, " Role conflict and ambiguity in complex organizations ", Administrative Science Quaterly, 15, 1970, p. 150-163.

ROUZIES D., BESSON M., " Le pilotage des forces de vente : effets pervers des systèmes hybrides ", Décisions Marketing, 14, 1998, p. 31-43.

SHARMA A., SAREL D., "The impact of customer satisfaction based incentives systems on salespeople's customer service response: an empirical study ", The Journal of Personal Selling Sales Management, 15, 1995, p. 17-29.

SPENCE M., « Job market signaling », Quarterly Journal of Economics, 1973.

WIDMIER S., "The effects of incentives and personality on salesperson's customer orientation ", Industrial Marketing Management, 31, 2002, p. 609-615.

ZAR J.H., Biostatistical Analysis, 4th edit, Prentice-Hall International publication, 1999.

Résumé : La plupart des téléconseillers ont dans la réalisation de leur métier deux principaux rôles qui sont de vendre des produits, mais également de satisfaire leurs clients. Or ces deux rôles sont souvent incompatibles et conduisent à une ambiguïté de rôle qui est source de stress. Cette étude teste l'hypothèse selon laquelle le montant de 
la prime d'objectifs dans le salaire de base affecte le degré d'ambiguïté de rôle que les téléopérateurs peuvent ressentir. Nous proposons que les primes d'objectifs transmettent des signaux sur l'importance relative de chacun de ces rôles. L'échantillon se compose de cent dix huit téléconseillers de sept centres d'appels. Le principal résultat est que la relation entre la part de la prime d'objectifs dans le salaire de base et l'ambiguïté de rôle est en U inversé.

Mots-clés : Centres d'appels, théorie des rôles, théorie du signal, prime d'objectifs, ambiguïté de rôle.

Abstract : Most teleoperators have two key roles: to sell products and satisfy customers. These two roles are often incompatible, and the resulting role ambiguity causes job stress. This study tests the hypothesis that the amount of bonus as a percentage of base salary affects the degree of role ambiguity that teleoperators experience. We assumed that bonuses transmit messages about the relative importance of job roles. The sample consisted of 118 teleoperators in seven call centers. The main result is that the relationship between the amount of bonus as a percentage of base salary and role ambiguity is an inverted- $U$.

Keywords : Call centers, role theory, signaling theory, bonuses, role ambiguity. 


\section{ANNEXE 1 : Echantillon de l'étude exploratoire.}

\begin{tabular}{|l|l|}
\hline \multicolumn{1}{|c|}{ Entretien } & \multicolumn{1}{|c|}{ Caractéristiques du téléconseiller interviewé } \\
\hline Entretien $n^{\circ} 1$ & Femme -45 ans \\
\hline Entretien $n^{\circ} 2$ & Homme -28 ans \\
\hline Entretien $n^{\circ} 3$ & Femme -23 ans \\
\hline Entretien $n^{\circ} 4$ & Homme -26 ans \\
\hline Entretien $n^{\circ} 5$ & Femme -35 ans \\
\hline Entretien $n^{\circ} 6$ & Homme -25 ans \\
\hline Entretien $n^{\circ} 7$ & Femme -42 ans \\
\hline Entretien $n^{\circ} 8$ & Femme -29 ans \\
\hline Entretien $n^{\circ} 9$ & Femme -39 ans \\
\hline Entretien $n^{\circ} 10$ & Homme -33 ans \\
\hline Entretien $n^{\circ} 11$ & Femme -21 ans \\
\hline Entretien $n^{\circ} 12$ & Homme -30 ans \\
\hline Entretien $n^{\circ} 13$ & Homme -31 ans \\
\hline Entretien $n^{\circ} 14$ & Femme -24 ans \\
\hline Entretien $n^{\circ} 15$ & Homme -46 ans \\
\hline Entretien & Caractéristiques du responsable d'équipe interviewé \\
\hline Entretien $n^{\circ} 16$ & Homme -49 ans \\
\hline Entretien $n^{\circ} 17$ & Femme -32 ans \\
\hline Entretien $n^{\circ} 18$ & Femme -43 ans \\
\hline Entretien $n^{\circ} 19$ & Homme -49 ans \\
\hline Entretien $n^{\circ} 20$ & Homme -39 ans \\
\hline
\end{tabular}

Tableau 5 : Caractéristiques des téléconseillers et responsables d'équipe interviewés lors de la phase exploratoire 


\section{ANNEXE 2 : Échelles de mesure}

\section{Montant de la prime d'objectifs et montant du salaire fixe :}

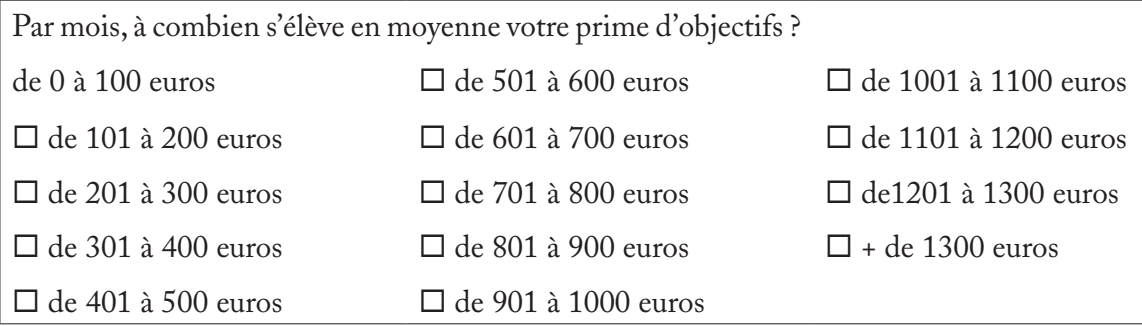

Une échelle de Likert à cinq points ( 1 = pas du tout d'accord, 5 = tout à fait d'accord) a été retenue.

Item 1 : Je connais avec certitude mon étendue d'autorité.

Item 2 : J'ai pour effectuer mon travail des objectifs clairs et planifiés.

Item 3 : Je sais que j'ai correctement réparti mon temps de travail.

Item 4 : Je sais quelles sont mes responsabilités.

Item 5 : Je sais exactement ce que l'on attend de moi.

Items 6 : Les explications sur ce qui doit être fait sont claires. 
